\title{
Factors Associated with Congenital Heart Diseases Among Children in Uganda: A Case-Control Study at Mulago National Referral Hospital (Uganda Heart Institute)
}

\author{
Grace Kahambu Kapakasi ${ }^{1}$, Ratib Mawa ${ }^{2,}$, , Judith Namuyonga ${ }^{3,4}$, Sulaiman Lubega ${ }^{3,4}$ \\ ${ }^{1}$ Department of Nursing, Victoria University, Kampala, Uganda \\ ${ }^{2}$ Department of Public Heath, Victoria University, Kampala, Uganda \\ ${ }^{3}$ Division of Paediatric Cardiology, Uganda Heart Institute, Kampala, Uganda \\ ${ }^{4}$ Department of Paediatrics and Child Health, Makerere University, Kampala, Uganda
}

Email address:

kapakasigracianne@gmail.com (G. K. Kapakasi), mawaratib2016@gmail.com (R. Mawa), jnamuyonga@gmail.com (J. Namuyonga), lubsk2001@yahoo.com (S. Lubega)

${ }^{*}$ Corresponding author

\section{To cite this article:}

Grace Kahambu Kapakasi, Ratib Mawa, Judith Namuyonga, Sulaiman Lubega. Factors Associated with Congenital Heart Diseases Among Children in Uganda: A Case-Control Study at Mulago National Referral Hospital (Uganda Heart Institute). Cardiology and Cardiovascular Research. Vol. 5, No. 1, 2021, pp. 1-6. doi: 10.11648/j.ccr.20210501.11

Received: September 24, 2020; Accepted: October 23, 2020; Published: January 12, 2021

\begin{abstract}
Congenital Heart Diseases (CHD) are among the leading causes of morbidity and mortality associated with congenital malformations among children. Not knowing the risk profile of CHD among children in Uganda impedes development of effective prevention interventions. In this hospital based unmatched case-control study we examined risk factors for all types of CHD among 179 pair of case and control children aged 0-10 years old at Mulago National Referral Hospital. Odds ratios and their corresponding 95\% confidence intervals were calculated using multivariate logistic regression. Low birth weight (adjusted OR: 3.15, 95\% CI 1.48 - 6.69), high birth order $\geq 5^{\text {th }}$ birth order (adjusted OR: 3.69 (1.10 - 12.54), maternal febrile illness during pregnancy, maternal and paternal alcohol consumption, and paternal socio-economic status were associated with CHD. Family history of CHD, maternal education level, maternal chronic illness, and paternal education level were not associated with CHD. The results suggest: low birth weight, high birth order, and maternal febrile illness during pregnancy, parental alcohol use and paternal socio-economic status as dominant risk factors for CHD among children. Rigorous implementation of public health policies and strategies targeting prevention of febrile illness during pregnancy, maternal malnutrition, parental alcohol consumption, delivery of high number of children per woman, might be important in reducing the burden of CHD among children in Uganda.
\end{abstract}

Keywords: Risk Factors, Alcohol Use, Low Birth Weight, Maternal Alcohol Consumption, Congenital Heart Diseases, Children, Uganda

\section{Introduction}

Congenital heart diseases (CHD) are among the leading causes of congenital malformation morbidity, and mortality and developmental delays among children across the globe [1, 2]. In 2010, an estimated 302,000 infants died of CHD. This accounted for $6 \%$ of all infant mortality across the globe, with $96 \%$ of these deaths estimated to have occurred in developing countries [3]. Although an estimated 34.5\% decline in CHD specific mortality among children was registered between 1990 and 2017 across the globe [1], the global prevalence of CHD among children remained high, i.e. 9.41 per 1000 live births, with marked variations across global geographical regions [4]. In the African region the prevalence of CHD was estimated at 2.32 per 1000 live births lower than that of other regions of the globe [4]. However this might be an under-estimate, because of low CHD diagnostic capacity, and early death of children with CHD before its diagnosis [5]. In Uganda, recent estimates indicated that approximately 8300 children are born with CHD every 
year, and over 2000 of these cases are severe enough to necessitate surgical treatment [6].

The causal model for CHD provides the theoretical grounding for this study [7]. Causes of CHD remain largely unknown, though approximately $15 \%$ the cases are linked to known causes such as chromosomal aneuploidies and defects in a single gene [8]. Epidemiological risk factors associated with CHD among children include but not limited to advanced maternal reproductive age, paternal age of $>25$ years [9], maternal chronic conditions such as diabetes mellitus [4], hypertension [10], and overweight and obesity [11], family history of CHD, previous still birth, maternal febrile illness during pregnancy, threatened abortion [12], parental alcohol use [13], parental tobacco use [14], maternal exposure to polycyclic aromatic hydrocarbon [15] and last but not least, consanguineous marriages [16].

Despite of the enormous epidemiological evidence on risk factors for CHD among children, no single study known to us examined the risk factors for CHD among children in Uganda. Recently published epidemiological studies in Uganda focused on description of the burden of CHD among children and adults, and the narrative of history of establishment CHD surgical treatment interventions [17-19]. Understanding the epidemiological risk profile for CHD among children in Uganda, is essential for developing prevention interventions that will contribute towards the realization of the vision of Uganda Heart Association [19], and the achievement of the sustainable development goal number 3.2 of reducing under-5 mortality to 25 deaths per 1,000 live births by the year 2030 [20]. The aim of this study is to examine the relationship between CHD and the following risk factors: parental alcohol use, maternal obstetric history and medical conditions and socio-demographic characteristics among children in Uganda.

\section{Methods and Materials}

\subsection{Study Design and Setting}

An unmatched case-control study conducted at Uganda Heart Institute (UHI), Mulago National Referral Hospital located approximately 5 kilometers from Kampala Capital City`s business center.

\subsection{Study Population}

A total of 358 (179 cases and 179 controls) children aged 0-10 years selected from outpatient treatment services at Mulago National Referral Hospital through consecutive sampling method were investigated. A case was defined as a child aged 0-10 years old, diagnosed with any type of CHD through transthoracic echocardiography by Paediatric Cardiologists using a Sonos 5500 (Philips, Best, Netherlands) and a Philips IE 33 (Philips, Best, Netherlands). Controls were defined as children of the same age range as cases clinically diagnosed free of CHD by Pediatricians. One control per case was selected through consecutive sampling without matching. All cases and controls were Ugandans by nationality.

\subsection{Exposure Assessment}

A pre-tested questionnaire dispensed through face-to-face interview was used to collect the data on the following exposure variables: child's size at birth, birth order, history of maternal febrile illness during pregnancy, history of maternal chronic diseases, maternal and paternal alcohol consumption, paternal occupation and education level, and other covariates such as: child's age in months, maternal age at conception, maternal education level, and family history of CHD.

\subsection{Statistical Analysis}

Comparison in distribution of cases and controls by sociodemographic, parental lifestyle and obstetric/maternal medical conditions were examined through cross-tabulation. Difference in distribution of cases and controls by categorical variables were tested using chi-square test. Conditional binary logistic regression model was fitted to calculate the odds ratios and their corresponding 95\% confidence intervals for CHD by each exposure variable. Statistical analysis was performed using SPSS version 23.

\subsection{Ethical Consideration}

The study adhered to Uganda national guidelines for research involving human participants [21]. Mothers to case and control children provided informed verbal consent. The study was approved by Mulago National Referral Hospital Research Ethics Committee (approval number MHREC 1593).

\section{Results}

Table 1, details the distribution of cases and controls by socio-demographic, maternal obstetric and medical-related conditions and parental lifestyle characteristics. Statistically significant differences were found between cases and controls with regards to sex, age, size at birth (proxy measure of birth weight), paternal education and occupation, maternal alcohol use, maternal chronic illness, maternal febrile illness during pregnancy, paternal alcohol consumption, and paternal smoking but not birth order, maternal age at conception, maternal education level and maternal occupation. 
Table 1. Distribution of case and control children by socio-demographic, parental lifestyle and medical characteristics.

\begin{tabular}{|c|c|c|c|c|}
\hline \multirow{2}{*}{ Characteristics } & \multirow{2}{*}{$\begin{array}{l}\text { Cases, } \mathbf{n = 1 7 9} \\
\mathrm{N}(\%)\end{array}$} & \multirow{2}{*}{$\begin{array}{l}\text { Controls, } \mathbf{n}=179 \\
\mathrm{~N}(\%)\end{array}$} & \multirow{2}{*}{$\begin{array}{l}\text { Total, } \mathbf{n}=\mathbf{3 5 8} \\
\mathbf{N}(\%)\end{array}$} & \multirow{2}{*}{ p-Value } \\
\hline & & & & \\
\hline \multicolumn{5}{|l|}{ Child`s sex } \\
\hline Female & $99(55.30)$ & $74(41.30)$ & $173(48.30)$ & \multirow{3}{*}{0.01} \\
\hline Male & $80(44.70)$ & $105(58.70)$ & $185(51.70)$ & \\
\hline \multicolumn{4}{|l|}{ Children's age (Months) } & \\
\hline $0-12$ & $92(51.40)$ & $43(24.00)$ & $135(37.70)$ & \\
\hline $13-36$ & $40(22.30)$ & $47(26.30)$ & $87(24.30)$ & \multirow{4}{*}{$<0.001$} \\
\hline $37-60$ & $23(12.80)$ & $26(14.50)$ & $49(13.70)$ & \\
\hline $61-84$ & $10(5.60)$ & $37(20.70)$ & $47(13.10)$ & \\
\hline $85-120$ & $14(7.80)$ & $26(14.50)$ & $40(11.20)$ & \\
\hline \multicolumn{5}{|l|}{ Child`s size at birth } \\
\hline Very small/Small & $62(34.60)$ & $18(10.10)$ & $80(22.30)$ & \multirow[b]{2}{*}{$<0.001$} \\
\hline Average/Large/Very large & $117(65.40)$ & $161(89.90)$ & $278(77.70)$ & \\
\hline \multicolumn{5}{|l|}{ Birth order } \\
\hline $1^{\text {st }}$ Born & $49(27.40)$ & $57(31.80)$ & $106(29.60)$ & \multirow{5}{*}{0.31} \\
\hline $2^{\text {nd }}$ & $51(28.50)$ & $55(30.70)$ & $106(29.60)$ & \\
\hline $3^{\text {rd }}$ & $32(17.90)$ & $34(19.00)$ & $66(18.40)$ & \\
\hline $4^{\text {th }}$ & $19(10.60)$ & $18(10.10)$ & $37(10.30)$ & \\
\hline $5^{\text {th }}$ and above & $28(15.60)$ & $15(8.40)$ & $43(12.00)$ & \\
\hline \multicolumn{5}{|l|}{ Maternal Age at conception (years) } \\
\hline $16-20$ & $18(10.10)$ & $20(11.20)$ & $38(10.60)$ & \multirow{5}{*}{0.84} \\
\hline $21-25$ & $58(32.40)$ & $60(33.50)$ & $118(33.00)$ & \\
\hline $26-30$ & $52(29.10)$ & $43(24.00)$ & $95(26.50)$ & \\
\hline $31-35$ & $35(19.60)$ & $36(20.10)$ & $71(19.80)$ & \\
\hline $36-45$ & $16(8.90)$ & $20(11.20)$ & $36(10.10)$ & \\
\hline \multicolumn{5}{|l|}{ Maternal Education Level } \\
\hline No education/Primary & $46(25.70)$ & $56(31.30)$ & $102(28.50)$ & \multirow[b]{3}{*}{0.49} \\
\hline Secondary & $77(43.00)$ & $73(40.80)$ & $150(41.90)$ & \\
\hline Tertiary & $56(32.30)$ & $50(27.90)$ & $106(29.60)$ & \\
\hline \multicolumn{5}{|l|}{ Paternal Education level } \\
\hline Primary & $13(21.70)$ & $47(78.30)$ & $60(16.90)$ & \multirow{3}{*}{$<0.001$} \\
\hline Secondary & $66(51.20)$ & $63(48.80)$ & $129(36.60)$ & \\
\hline Tertiary & $96(58.20)$ & $69(41.80)$ & $165(46.60)$ & \\
\hline \multicolumn{5}{|l|}{ Paternal Occupation } \\
\hline Not working & $10(5.60)$ & $10(5.70)$ & $20(5.60)$ & \\
\hline Professional/Technical/Managerial & $94(37.60)$ & $59(26.70)$ & $153(42.90)$ & $<0.01$ \\
\hline Secretarial/Clerical/Skilled manual & $36(19.10)$ & $66(10.80)$ & $102(28.60)$ & \\
\hline Unskilled manual/Farmer & $38(37.60)$ & $44(56.80)$ & $82(23.0)$ & \\
\hline Family history of CHD (any of the & & & & \\
\hline Yes & $22(12.40)$ & $9(5.00)$ & $31(8.70)$ & 0.01 \\
\hline No & $156(87.60)$ & $170(95.000)$ & $326(91.30)$ & \\
\hline Maternal Alcohol Consumption & & & & \\
\hline Yes & $65(36.30)$ & $12(6.70)$ & $77(21.50)$ & \\
\hline No & $114(63.70)$ & $167(93.30)$ & $281(78.50)$ & $<0.001$ \\
\hline Mother had chronic illness before $p$ & & & & \\
\hline Yes & $25(14.00)$ & $10(5.60)$ & $35(9.80)$ & \\
\hline No & $154(86.00)$ & $169(94.40)$ & $323(90.20)$ & 0.01 \\
\hline Mother had febrile illness during pr & & & & \\
\hline Yes & $79(44.10)$ & $16(8.90)$ & $95(26.50)$ & \\
\hline No & $100(55.90)$ & $163(91.10)$ & $263(73.50)$ & $<0.001$ \\
\hline Paternal alcohol consumption & & & & \\
\hline Yes & $108(60.30)$ & $32(17.90)$ & $140(39.10)$ & \\
\hline No & $71(39.70)$ & $147(82.10)$ & $218(60.90)$ & $<0.001$ \\
\hline
\end{tabular}

Table 2 shows the crude and adjusted odds ratios and their corresponding $95 \%$ confidence intervals for CHD among children by risk factors investigated. In the adjusted model, children who had very small/small size at birth (proxy measure of birth weight) had higher risk of CHD compared to those who had average/above average size at birth. Likewise the risk of CHD among children increased with increase in birth order with those born in $\geq 5^{\text {th }}$ birth order having the highest risk of CHD compared to those in the first birth order. On the same note, the results also showed high risk of CHD among children who had family history of CHD compared to those without. Maternal febrile and chronic illnesses during pregnancy also increased the risk of CHD in their offspring compared to those whose mothers didn't experience these illnesses. The risk of CHD also tended to increase with increase in maternal age at conception, however the highest risk of CHD was recorded among children whose mothers were in age range of 21-25 years at conception relative to those in the oldest age group of 36-45 years. High risk of CHD was also found among children 
whose parents drink alcohol compared to those whose parents don't drink alcoholic beverages. Our results also showed that the risk of CHD among children tended to increase with increase in paternal socio-economic status (social status) measured using education level and occupation.
Lastly children whose mothers had primary and secondary level education were found to have higher risk of CHD compared to those whose mothers had tertiary level education.

Table 2. Crude and adjusted odds ratio (OR) and 95\% confidence interval (CI) for CHD by socio-demographic, parental lifestyle and medical characteristics.

\begin{tabular}{|c|c|c|}
\hline Characteristic & Crude OR (95\% CI) & Adjusted OR (95\% CI) \\
\hline \multicolumn{3}{|l|}{ Child`s size at birth } \\
\hline Average/above average & 1.00 & 1.00 \\
\hline Very small/Small & $4.74(2.66-8.43)$ & $3.15(1.48-6.69)$ \\
\hline \multicolumn{3}{|l|}{ Birth Order } \\
\hline $1^{\text {st }}$ & 1.00 & 1.00 \\
\hline $2^{\text {nd }}$ & $1.08(0.63-1.85)$ & $1.36(0.62-3.03)$ \\
\hline $3^{\text {rd }}$ & $1.09(0.59-2.03)$ & $1.90(0.66-5.45)$ \\
\hline $4^{\text {th }}$ & $1.23(0.58-2.59)$ & $1.57(0.48-5.16)$ \\
\hline$\geq 5^{\text {th }}$ & $2.17(1.04-4.53)$ & $3.69(1.10-12.54)$ \\
\hline \multicolumn{3}{|l|}{ Family history of CHD } \\
\hline No & 1.00 & 1.00 \\
\hline Yes & $2.67(1.19-5.96)$ & $1.75(0.51-5.98)$ \\
\hline \multicolumn{3}{|l|}{ Maternal Education Level } \\
\hline Tertiary & 1.00 & 1.00 \\
\hline Primary & $0.73(0.42-1.26)$ & $2.38(0.88-6.46)$ \\
\hline Secondary & $0.94(0.57-1.55)$ & $1.76(0.79-3.89)$ \\
\hline \multicolumn{3}{|l|}{ Maternal age at conception (Years) } \\
\hline $36-45$ & 1.00 & 1.00 \\
\hline $16-20$ & $1.13(0.45-2.80)$ & $1.03(0.22-4.81)$ \\
\hline $21-25$ & $1.21(0.57-2.56)$ & $2.01(0.57-7.04)$ \\
\hline $26-30$ & $1.51(0.70-3.27)$ & $1.90(0.61-5.96)$ \\
\hline $31-35$ & $1.22(0.54-2.72)$ & $1.10(0.34-3.52)$ \\
\hline \multicolumn{3}{|l|}{ Maternal alcohol consumption } \\
\hline No & 1.00 & 1.00 \\
\hline Yes & $7.93(4.10-15.36)$ & $3.12(1.24-7.87)$ \\
\hline \multicolumn{3}{|c|}{ Maternal chronic illness during pregnancy } \\
\hline No & 1.00 & 1.00 \\
\hline Yes & $2.74(1.28-5.89)$ & $0.44(0.16-1.21)$ \\
\hline \multicolumn{3}{|c|}{ Maternal Febrile illness during pregnancy } \\
\hline No & 1.00 & 1.00 \\
\hline Yes & $8.05(4.45-14.55)$ & $7.75(3.75-15.97)$ \\
\hline \multicolumn{3}{|l|}{ Paternal alcohol consumption } \\
\hline No & 1.00 & 1.00 \\
\hline Yes & $6.99(4.10-11.36)$ & $4.09(2.08-8.03)$ \\
\hline \multicolumn{3}{|l|}{ Paternal education level } \\
\hline Tertiary & 1.00 & 1.00 \\
\hline Primary & $0.20(0.10-0.39)$ & $0.41(0.09-1.71)$ \\
\hline Secondary & $0.75(0.47-1.19)$ & $2.11(0.69-6.49)$ \\
\hline \multicolumn{3}{|l|}{ Paternal Occupation } \\
\hline Unskilled manual/Farmer & 1.00 & 1.00 \\
\hline Not working & $1.59(0.62-4.06)$ & $2.56(0.57-11.49)$ \\
\hline Professional/Managerial & $0.55(0.21-1.43)$ & $4.92(1.54-15.71)$ \\
\hline Secretarial/Clerical/Skilled manual & $0.86(0.33-2.30)$ & $5.75(1.85-17.88)$ \\
\hline
\end{tabular}

Mutually adjusted for each other.

\section{Discussion}

Our results showed three-fold increase in the risk of all CHD among children who were small at birth (low birth weight), similar to results of an Indian hospital based casecontrol study [22]. Low birth weight might be linked to maternal malnutrition during pregnancy, known to affect organogenesis [23].

Children's birth order was associated with CHD with an increasing trend in the risk of CHD by birth order (Table 2). However children in $\geq 5^{\text {th }}$ birth order were found to have four-fold increase in the risk of CHD relative to those in the $1^{\text {st }}$ birth order, similar to results of a Tanzanian crosssectional study of all congenital anomalies [24]. The sudden jump in odds ratio after $4^{\text {th }}$ birth order might be linked to inclusion of cases and controls in $\geq 5^{\text {th }}$ birth order in the same birth order category to fulfill logistic regression modeling condition that requires at least ten observations in each subgroup of a variable.

Maternal febrile illness during pregnancy was strongly associated with seven-fold increase in the risk of CHD, consistent with results of a recent systematic review and 
meta-analysis that found a pooled odds ratio of 1.53 for CHD among children whose mothers experienced febrile illness during pregnancy, even though the later has small magnitude of the effect measure [25]. Febrile illnesses are linked to changes in uterine environment creating teratogenic substances that are implicated for occurrence of CHD among children [26].

Maternal chronic illness before and during pregnancy was found to have two-fold increase in the risk of CHD among children, but inadequate evidence was found to demonstrate existence of an association with $\mathrm{CHD}$, contrary to findings of a systematic review which showed a strong association between CHD and maternal diabetes [4], overweight and obesity [11] and maternal hypertension [10]. Lack of evidence of association between CHD and maternal chronic illness in our study might be linked to the effect of small sample size and confounders that might not be accounted for in the study.

Regarding parental lifestyle characteristics, maternal alcohol intake was associated with three-fold increase in the risk of CHD, similar to the results of a systematic review and meta-analysis [13]. Contrary to this finding is the results of a meta-analysis study that demonstrated no evidence of an association between maternal alcohol use and CHD among children [27], creating inconsistency that needs further investigation.

Paternal alcohol consumption was found to be associated with four-fold increase in the risk of CHD, similar to the results of a recent meta-analysis [13]. Imaging techniques and optical coherence tomography study of embryos revealed that changes in heart function due to ethanol exposure tend to appear before structural changes occur, and concluded that structural heart changes might occur as a result of effects of changes in heart function [28].

Lastly, in this study we found conflicting results with regards to the relationship between CHD and socio-economic status of parents. Although, the risk of CHD increased with decrease in maternal education level, and with increase in paternal education level, we didn't find evidence of an association between either maternal education or paternal education and CHD among their offspring. However when paternal socio-economic status was measured using occupation, a strong positive association was found between paternal socio-economic status and CHD among children. This inconsistencies might suggest that education might not be a suitable measure of socio-economic status in Ugandan setting. However our findings are consistent with results of a meta-analysis which found a no association between maternal socio-economic status, (measured using education level) and CHD among children [29] and those of a Cohort study conducted in California (US) that found an association between social deprivation and increased risk of CHD among children, to which authors attributed to environmental exposure of deprived communities to pollutants [30].

\section{Conclusion}

Low birth weight, higher birth order $(\geq 5)$, maternal febrile infections during pregnancy, maternal, and paternal alcohol consumption and paternal socio-economic status are associated with CHD among children. Inadequate evidence was found to conclude existence of a relationship between CHD and family history of CHD, maternal education level, maternal history of chronic illness and paternal education level. However these findings should be interpreted with caution, because retrospective exposure assessment might have resulted into recall bias and exposure misclassification with respect to the outcome (CHD). Like-wise the small sample size might have affected the precision and magnitude of the effect measure for each risk factor. Interventions targeting these modifiable risk factors might be instrumental in reducing the burden of CHD among children in Uganda. Further studies are required to explore dose-response relationship between paternal alcohol use and CHD, maternal chronic illness types and CHD, maternal alcohol consumption and CHD, specific febrile illness during pregnancy and CHD as well as examination of factors behind parental socio-economic differences and the occurrence of CHD among children.

\section{Conflict of Interest}

The authors declare no conflict of interest.

\section{Acknowledgement}

We wish to acknowledge the management of Uganda Heart Institute and Mulago National Referral Hospital for providing the opportunity to conduct this study and Prof Lawoko Stephen for his ideas and contribution in critiquing the research questions at the conceptualization stage.

\section{References}

[1] Zimmerman, M. S., et al., Global, regional, and national burden of congenital heart disease, 1990-2017: a systematic analysis for the Global Burden of Disease Study 2017. The Lancet Child \& Adolescent Health, 2020. 4 (3): p. 185-200.

[2] Mari, M. A., M. M. Cascudo, and J. C. Alchieri, Congenital heart disease and impacts on child development. Brazilian journal of cardiovascular surgery, 2016. 31 (1): p. 31-37.

[3] Bernier, P.-L., et al. The challenge of congenital heart disease worldwide: epidemiologic and demographic facts. in Seminars in Thoracic and Cardiovascular Surgery: Pediatric Cardiac Surgery Annual. 2010. Elsevier.

[4] Chen, L., et al., Risk of congenital heart defects in offspring exposed to maternal diabetes mellitus: an updated systematic review and meta-analysis. Archives of gynecology and obstetrics, 2019: p. 1-16.

[5] Jivanji, S. G., et al., Congenital Heart Disease in East Africa. Frontiers in pediatrics, 2019. 7.

[6] Aliku, T. O., et al., Pediatric cardiovascular care in Uganda: Current status, challenges, and opportunities for the future. Annals of pediatric cardiology, 2017. 10 (1): p. 50. 
[7] Gelb, B. D., History of our understanding of the causes of congenital heart disease. Circulation: Cardiovascular Genetics, 2015. 8 (3): p. 529-536.

[8] Erikssen, G., et al., Achievements in congenital heart defect surgery: a prospective, 40-year study of 7038 patients. Circulation, 2015. 131 (4): p. 337-346.

[9] Abqari, S., et al., Profile and risk factors for congenital heart defects: A study in a tertiary care hospital. Annals of pediatric cardiology, 2016.9 (3): p. 216.

[10] Ramakrishnan, A., et al., Maternal hypertension during pregnancy and the risk of congenital heart defects in offspring: a systematic review and meta-analysis. Pediatric cardiology, 2015. 36 (7): p. 1442-1451.

[11] Persson, M., et al., Maternal overweight and obesity and risk of congenital heart defects. Journal of the American College of Cardiology, 2019.73 (1): p. 44-53.

[12] Ou, Y., et al., Risk factors of different congenital heart defects in Guangdong, China. Pediatric research, 2016. 79 (4): p. 549.

[13] Zhang, S., et al., Parental alcohol consumption and the risk of congenital heart diseases in offspring: An updated systematic review and meta-analysis. European Journal of Preventive Cardiology, 2020. 27 (4): p. 410-421.

[14] Zhao, L., et al., Parental smoking and the risk of congenital heart defects in offspring: an updated meta-analysis of observational studies. European journal of preventive cardiology, 2019: p. 2047487319831367.

[15] Liu, X., et al., Does maternal environmental tobacco smoke interact with social-demographics and environmental factors on congenital heart defects? Environmental Pollution, 2018. 234: p. 214-222.

[16] Ahmadi, A., et al., Risk factors of congenital heart diseases: A hospital-based case-control study in Isfahan, Iran. ARYA Atherosclerosis, 2020. 16 (1): p. 1-6.

[17] Caddell, J. and D. Connor, Congenital heart disease in Ugandan children. British heart journal, 1966. 28 (6): p. 766.

[18] Namuyonga, J., et al., High prevalence of truncus arteriosus in pediatric congenital heart disease in Uganda. Annals of pediatric cardiology, 2019. 12 (2): p. 186.
[19] Lwabi, P., et al., The Uganda Heart Association: Developing cardiovascular care for the 45 million population is the objective of the Uganda Heart Association. 2019, Oxford University Press.

[20] Lee, B. X., et al., Transforming our world: implementing the 2030 agenda through sustainable development goal indicators. Journal of public health policy, 2016. 37 (1): p. 13-31.

[21] Science, U. N. C. f. and Technology, National guidelines for research involving humans as research participants. 2014: Uganda National Council for Scince and Technology Kampala-Uganda.

[22] Haq, F. U., et al., Risk factors predisposing to congenital heart defects. Annals of pediatric cardiology, 2011. 4 (2): p. 117.

[23] Godfrey, K. M. and D. J. Barker, Fetal programming and adult health. Public health nutrition, 2001. 4 (2b): p. 611-624.

[24] Mashuda, F., et al., Pattern and factors associated with congenital anomalies among young infants admitted at Bugando medical centre, Mwanza, Tanzania. BMC research notes, 2014.7 (1): p. 1-7.

[25] Shi, Q., et al., Congenital heart defects and maternal fever: systematic review and meta-analysis. Journal of Perinatology, 2014. 34 (9): p. 677-682.

[26] Kalisch-Smith, J. I., N. Ved, and D. B. Sparrow, Environmental Risk Factors for Congenital Heart Disease. Cold Spring Harbor Perspectives in Biology, 2020. 12 (3): p. a037234.

[27] Yang, J., et al., Prenatal alcohol exposure and congenital heart defects: a meta-analysis. PloS one, 2015. 10 (6).

[28] Karunamuni, G., et al., Ethanol exposure alters early cardiac function in the looping heart: a mechanism for congenital heart defects? American Journal of Physiology-Heart and Circulatory Physiology, 2014. 306 (3): p. H414-H421.

[29] Yu, D., et al., Maternal socioeconomic status and the risk of congenital heart defects in offspring: a meta-analysis of 33 studies. PLoS One, 2014. 9 (10): p. e111056.

[30] Peyvandi, S., et al., Environmental and Socioeconomic Factors Influence the Live-Born Incidence of Congenital Heart Disease: A Population-Based Study in California. Journal of the American Heart Association, 2020. 9 (8): p. e015255. 